\title{
A Survey on Routing Protocols influencing Mobile Sink in enhancing life time of WSN for Data gathering
}

\author{
${ }^{1}$ S.Suseela, ${ }^{2}$ S. Jeevanantham \\ 1Assistant Professor Department of Computer Science and Engineering, Peiryar Maniammai \\ UniversityThanjavur, TamilNadu, India \\ 2M.Tech Freelancer, Networking Consultant, Thanjavur
}

\begin{abstract}
Wireless Sensor Network is a group of specialized sensors have the ability to sense, monitor, communicate to the neighbors and recording conditions at assorted locations such as temperature, sound, pressure, security purpose etc. The sensed data's incurred for estimation and then forwarded to the neighbors. There are many challenging issues in WSN such as localization, synchronization, data aggregation, dissemination, database querying, architecture, middleware, security, power consuming, routing, abstractions and higher level algorithms. Maximizing the lifetime of energy dearth Wireless Sensor Networks is a very big challenging issue in the present context. An optimized solution is needed to increase the lifetime of the Wireless Sensor Networks. In this paper the depiction of mobile sinks with a choice of routing protocols is used in different scenarios to enhance the efficiency and life time of WSN.
\end{abstract}

Keywords: Wireless Sensor Networks, Routing protocols, Data gathering, Data Dissemination, node life, Mobile Sink, power efficiency, challenges.

\section{Introduction}

Wireless Sensor Network largely deployed and used in the assorted fields such as military, landslide area, Tsunami and in all polluted area. Wireless Sensor Network has applied in various fields to access the respective information including environmental monitoring and security surveillance purposes [1]. Each node of the sensor network consists of three Subsystems namely Sensing Subsystem, Processing Subsystem and Communication Subsystem. In Sensing Subsystem it senses the environment, Processing Subsystem is processed the sensed data and Communication System which is responsible for message exchange with neighboring sensor.

Sensor network consists of different types of sensors such as seismic thermal visual and infrared used to monitor the different ambient conditions like temperature, humidity pressure and motion of the object and its characteristics. Since that nodes, can be used in military, health, chemical processing and disaster relief scenarios. The advantages of WSNs like rapid deployment, fault tolerance, high precise monitoring and timely response, make, them widely deployed in unfriendly or even harsh environments, such as military and disaster surveillance, industrial product line monitoring, agricultural, wildlife observation, healthcare, and smart homes etc., [2]. Researchers have faced many challenges in Wireless Sensor Networks.

The challenges are Node deployment, Energy Management, Data routing methods, Node/link heterogeneity, fault tolerance, Network dynamics, transmission media, Connectivity, Coverage, data aggregation, Quality of Service. Though many challenges existing, power limited resource is the main and major challenge and it leads short network lifetime of WSN. Each sensor node of WSN have limited sensing region, processing power and energy.

The paper is organized as follows. The concept of mobile sink is given in the Section 2.Section3.describes the wireless sensor architecture with mobile sink. Routing Protocols in Mobile Sink for Data Gathering Units are discussed in Section 4.Comparision result of routing protocol are presented in Section 5.Finally, the paper is concluded.

\section{Mobile Sink}

The Sink can be static or mobile and it can be placed at different location in the WSN. In the case of static sink, nodes located in the vicinity of the sink deplete their energy (and die) much earlier compared to the nodes located farther away from the sink moves along certain path through the network. The stationary sink node hop by hop, the sensor node near the sink have to consume more energy on forwarding data for other nodes which probably causes energy loss of the network. Using mobile sink can alleviate the hotspot problem and balance the energy consumption among the sensor nodes.However, sensor nodes are usually of powered with limited batteries and replacing or recharging batteries on a large scale is not realistic. In order to overcome this issue, sink mobilization has been introduced. Mobile sink moves along to a certain path through the network.Now a day's many energy efficient routing algorithms or protocols have been proposed with techniques 
like clustering, data fusion, multi-path, location tracking and sink mobility. Using sink mobility technologies avoids excessive transmission overhead at sensor nodes, since those sensors can forward their traffic only, when sink node is nearby.

Energy consumption can also get balanced since the hotspot nodes will rotate as sink nodes move around throughout the sensor network, and this will cause prolonged network lifetime. Besides, sink mobility technologies can ensure good network connectivity under sparse or disconnected sensor networks. Sink is one of the important components of a WSN and it acts as a translator between the sensor nodes and the end-user. Mobile sink has many advantages such as increasing the security of WSNs. Since the location of the mobile sink changes over time, the location of the mobile sink is hard to find for the malicious users andcan't damage it. Therefore the mobile sink may be used in security applications, such as medical care, target tracking and intrusion detection in battlefield. Instead of using static sink, mobile sink is able to move, sensor nodes can communicate with the sink, when it is near to them, and so hop by hop delivery path of data becomes while shorter. Mobile sink even can move close to nodes the power consuming of node is reduced. So the set of nodes near the mobile sink which provide more balance of energy. Hence the lifetime of network can be increased [3].

\section{Wireless Sensor Network Architecture With Mobile Sink}

The Figure.1 shows the example of WSN architecture. Sensors read and send information as data to the data sink.

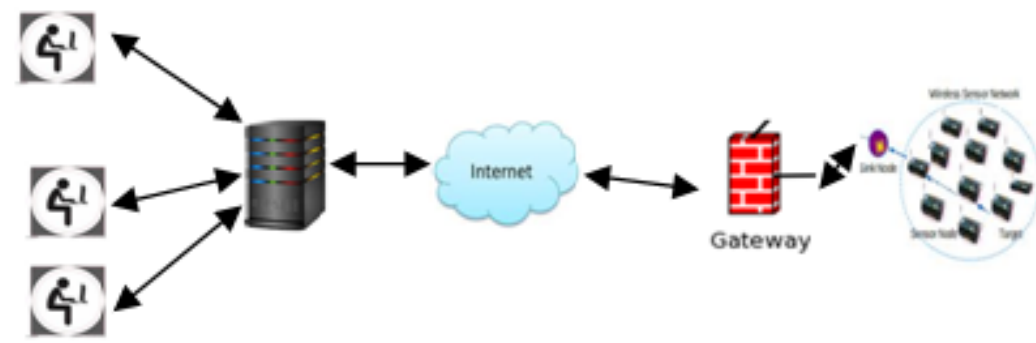

Figure.1. WSN Architecture

The sink acts as a gateway between the WSN and the end-user application. The basic approach is collecting data from sensor nodes, the process is direct one in which each sensor node transmit data directly to the sink even they are located far away. The expenditure of data transmission from each sensor node to the sink is very high; hence the nodes die quickly by reducing the lifetime of the network. If ensure a few transmissions as possible it will leads efficient energy utilization.The Architecture influences the efficient network deployment also defines how to manipulate sensor nodes, BS and Sink in WSN. Based on architecture can classify the WSN into three categories.

- $\quad$ Layered Architecture

- Cluster Architecture

- Sensor Nodes with Mobile Sink Architecture

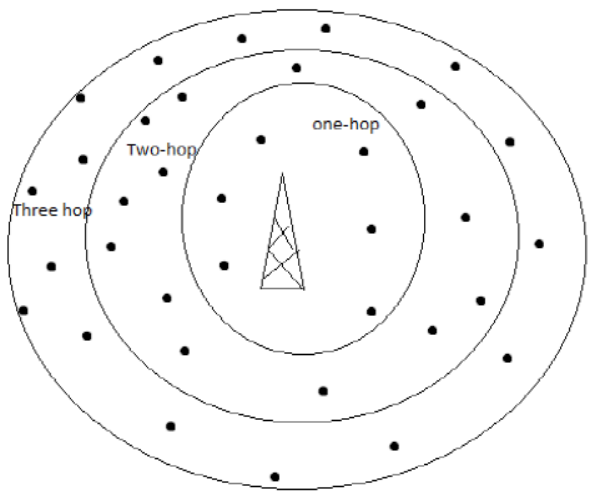

Figure.2. Layered Architecture

The Figure. 2 depicts a layered architecture; it consists of single sink node and multiple sensors node that sends the data to the sink using one hop, two hops and three hops etc. Sink node collects all information. 
The advantage of a layered architecture each node of the network is concerned about their short-distance, the architecture utilizes the low-power transmissions to nodes of the neighboring layers.

A group of sensor nodes forms the cluster and each cluster has the cluster head and a sink node shown in Figure.3.

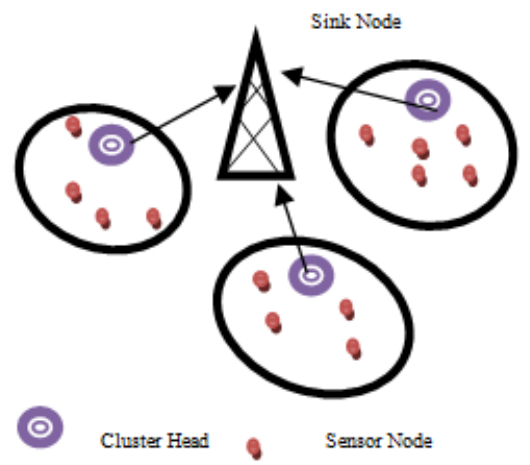

Figure.3. Clustered Architecture

The sink node receives the data's from the cluster head. The sink is normally called as BS. The clustering architecture provides two important features over the Layered architecture [4]. Firstly, clustered wireless sensor networks are capable of reducing the volume of inter-node communication by localizing data transmission within the formed clusters and decreasing the number of transmissions to the sink node; secondly, clustered wireless sensor networks are capable of extending the nodes' sleep times by allowing cluster heads to coordinate and optimize the activities.

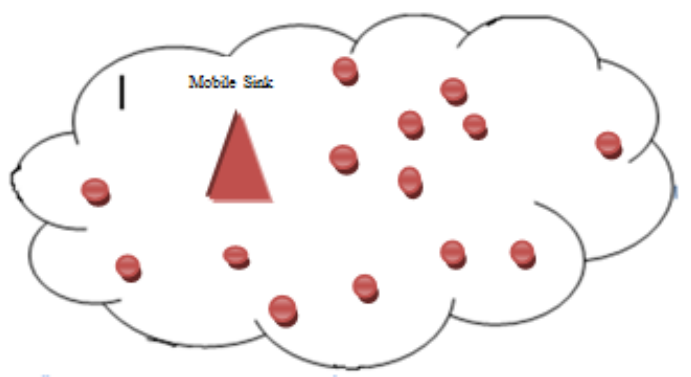

Figure.4. Sensor Nodes with Mobile Sink Architecture

In traditional static sink model usually multi hop technique is used to send the data. Since each and every node dissipates some energy while transfers the data, at some stage the node becomes to die. The rate of power dissipation depends on the distance from the sink and the number of times been accessed. So it is important to reduce the power consumption of the individual nodes. This issue could be alleviated through mobile sink. Sensor nodes with mobile sink architecture in Figure.4 consist of number of sensor nodes and few mobile sinks in which sink travels in sensing area and collects the data from sensor nodes.

\section{Routing Protocols In Mobile Sink For Data Gathering Units Wi-Fi}

Routing protocols play the vital role over communication paradigms that greatly affect the performance of the wireless sensor networks; so that designing routing protocols for sensor networks is a very necessary one. An efficient routing protocol with mobile sink helps to conserve the power. Existing work in designing routing protocol on mobile sinks can be classified in to three categories.

- One hop based strategy -The mobile sink is in their communication range for collecting data, the sinks move randomly or predefined paths

- Hierarchy based strategy : A the sensor nodes are formed as a cluster, a specified node in a cluster acts as a cluster head that collects the data and send to the mobile sink by someway

- Multihop based strategy : Sensornodes transmit their sensed data to mobile sinks by the means of multi-hop while the sinks are moving

Wireless Sensor Network is being used in many applications and sensors are used for variety of applications including battlefield surveillance, commercial application at home and industries, event detection such as intrusion/flood emergencies etc. Among all the applications one of the important role is data collection or data gathering. So gathering of data or collection of data has been approached in four ways [5]. 
- Discovery : Discovery is mobility independent and purely knowledge based

- Data Transfer: Data Transfer achieved through joint discovery

- Routing: Flat and proxy-based.

- Motion Control: Trajectory is both static or dynamic, speed and hybrid

Routing protocol supports mobile sinks to collect data in WSNs, even it has higher complexity and processing overhead yet they have encouraging features of the sink mobility especially in the low mobility regime [6].

- The mobility of sink is controllable and thus predictable.

- The pause time of a sink along its trajectory is much longer than the actual moving time.

- In general, existing routing protocols have some positive proactive features to cope with the dynamics in link quality, which can be broken, to support sink mobility.

\section{A. Mobiroute}

A static sink have higher protocol tocollect data in WSNs. However it leads to higher protocol complexity and overhead.

A routing protocol that supports mobile sinks to collect data in WSNs, compared with existing protocols for static WSN.However, the following favourable features of the sink mobility (in the low mobility regime) and of the existing routing protocols help,to limit the side effects:

- The mobility is controllable and thus predictable,

- The pause time of a sink along its moving trace is much longer than the actual moving time,

- Existing routing protocols usually possess some proactive features to cope up with the dynamics in link

- Qualitycan be exploited to support sink mobility.

The author engineered a routing protocol, which is working with the principle of discrete mobility pattern for a sink, which is often changing its location by time. MobiRoute is a superset of Berkeley MintRoute [7]. MintRoute concern to minimize the total number of transmission hence it uses a Minimum Transmission (MT) metric. Mt is to minimize the total number of transmissions (including retransmissions). Since the data rate is low, route messages do not need to be exchanged frequently. This helps MintRoute to reduce its energy consumption. The protocol could perform the following operation while transferring the data towards the sink.

- Node is notified when links getting broken with sink due to mobility.

- Disseminate to whole network about the topological

- Changes incurred by mobility.

- $\quad$ Packet loss is minimized during the sink moving period.

\section{B. Anchorbased Voronoi-scoping Routing Protocol (AVRP)}

Routing protocol design impacts the performance of an mWSN (Wireless Sensor Network with mobile Sinks). A complete protocol can increase the network efficiency while suppressing the protocol overhead due to sink mobility. AVRP design support the efficient data gathering in mWSN [8]. This protocol aimed for moderate and heavy traffic. It has ttwo features; one is Voronoi scoping [9], each sensor always delivers its data packets to its closest sink in a dynamic mWSN and the second one is Anchor node, anchor nodes are used to absorb the small movement of sinks while maintaining the good property of Voronoi scoping after creating forwarding structure. On the built delivery structure, each (mobile) sink only gathers data packets from the subset of sensor nodes within the sink's Voronoi cluster (or scope) in Figure.5. Scalability of mWSN is ensured by introducing dynamic selection of anchor nodes which are used to absorb the small movement of mobile sinks.

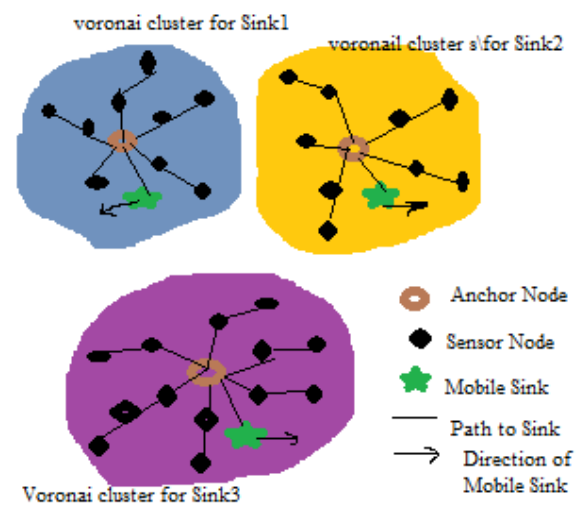

Figure.5. AVRP creates the anchor-based Voronoi scoping in an mWSN with three mobile sinks. 
There are two metrics used to define the efficiency of the network.

- Normalized forwarding overhead

- Packet delivery ratio

Normalized forwarding overhead $=$ Total Number of packets (data packets + control packets) transmitted / number of data packets delivered to all sinks.

Packet delivery ratio $=$ data packets received by all sinks / number of data packets transmitted by all sensor nodes.

\section{Mobile Sink based Routing Protocol (MSRP):}

MSRP protocol is designed to support and lengthen Network Lifetime of WSN since the structure must be in the form of cluster [10]. Sensed data transmitted from the nodes to Cluster Head (CH), it collects by mobile sink within its vicinity. Mobile sink along with data gathering it maintains $\mathrm{CH}$ residual energy information. The decision of sink movement based on the higher residual energy of $\mathrm{CH}$ sincethat the highest residual energy $\mathrm{CH}$ becomes the immediate neighbor of the mobile sink that minimize the hot spot problem. The result leads a balanced usage of energy and becomes the WSN have better life time.

\section{D.Energy-Efficient Mobile Sink Routing Algorithm (EEMSRA):}

In this algorithm cluster-based structured model used to implement the mobile sink routing protocol, and the sink moves based on the average energy in each cluster [11]. The algorithm has three phases.

- Initialization phase

- Data Transmitting phase

- Sink Moving phase

Initialization phase LEACH protocol used to cluster the WSN. Mobile sink have collected information from the cluster head $(\mathrm{CH})$ randomly. During Initialization phase, establish Distance Vector Table and compute time period according with distance vector table.In Data transmitting phase mobile sink moves to one $\mathrm{CH}$ and broadcast hello packet, Receiving $\mathrm{CH}$ store the time information and Cluster ID from the Hello packet. The node has the maximum energy will be selected as a new $\mathrm{CH}$. $\mathrm{CH}$ send the information sink message to the mobile sink when the time period is up; Sink Moving phase the mobile sink compares the energy parameter (Eavg) of the current cluster with the others received information from other cluster-heads: if the energy parameter (Eavg) is high than any of the received energy parameters (Eavg) from other cluster-heads, the mobile sink will not move. Otherwise the mobile sink will move to the cluster which has the maximum energy parameter (Eavg). This algorithm can do better performance for energy consumption.

\section{E. High-Reliability Data Gathering protocol based on Mobile Sinks (HRDG-MS):}

Recent years many methodologies about efficient data gathering in WSN are discussed on the science and technology forums as the frontline topics. Various protocols are introduced among all HRDG-MS is one of the protocol is highly reliable [12]. This protocol provides the adequate support to the mobile sinks, it can move randomly and sends Beacons signal periodically. At the same time the sensor nodes have the routes to mobile sinks send DATA packets which are incorporated with the state information of the nodes and their distance to mobile sinks. Assist of Beacons and DATA packets, the whole network is mapped and maintains the route information as routing table of traditional network without any route packet and location information.

Three node states have been defined in this HRDG-MS architecture one is AS state node, a node which is adjacent to mobile sink can send data directly to the sink in one hop. The second node state is FS state, the node far away from sink buthave route to the sink, so send the packet over multi hop. The third node state is IS state, the distance between mobile sink and the node is infinite, so there is no route to the sink. HRDG-MS is an overhearing-based protocol. The nodes with highest metric that is far distance forward their DATA packets to lower metric node till the packets are reached to mobile sinks by AS nodes shown in Figure.6

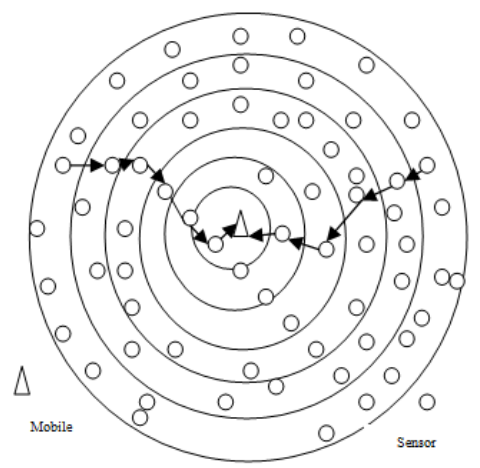

Figure. 6 The forwarding process of DATA packet in HRDG-MS 


\section{F. Energy Efficient Data Algorithm [MILP (Mixed Integer Linear Programming)]}

The advantage of using controlled sink mobility in clustered wireless sensor networks (WSNs) increases network lifetime. WSN clustered sensor network all Cluster Heads (CHs) have transmit their buffered data to the sink between some specified time interval is called data Heads ( $\mathrm{CHs})$ have transmitted their buffered data to the sink between some specified time intervals called date reporting time (tdr). MILP a mathematical model scheme proposed to collect all the CHs data in prescribed tdr time span [13]. This model have considered several constraints to minimize the maximum energy consumed by $\mathrm{CHs}$ such as data reporting time surrounded by $\mathrm{CHs}$, buffers size of $\mathrm{CHs}$ and energy. This algorithm determines the best sink path while each $\mathrm{CH}$ can freely change its transmission range (ri) in order to maximize network lifetime.

\section{G. Mobile Sink Based Reliable and Energy Efficient Data Gathering (MSREEDG)}

A technique used a tree based network topology which provides both the reliability and energy efficiency [14]. The technique has two steps, in which the optimal sink path estimation technique cost of the in network communication is estimated and as well sink's position estimation has done and reliable data transmission techniques involves threephases: data encoding, communication and data decoding. The biased random walk and rendezvous point selection method is used to determine the next position of the sink and the optimal data transmission path. In addition to it the sensor encodes the data by using RS coding and transmit to the mobile sink, then the mobile sink decodes the messages and reconstructs the original bundle.

The pause time depends on node density and received encoded data. That will improve the performance of the nodes. In wireless sensor network some data are duplicate or unnecessary which can be received by sensor nodes multiple times. Therefore, such surplus messages will increase the average energy consumption of network. So power constrained rechargeable battery resource is expected to run for a long time, since these limitation and expenses of energyare the issues are considered to be in data gathering [15], [16], [17].

\section{H. Mobile Sink Based Adaptive Immune Energy-EfficientClustering Protocol [MSIEEP]}

The sensor nodes near the static sink act as relays for distant sensors so it drain their energy very quickly, resulting energy holes in the sensor field. The energy holes problem will lead the premature disconnection of the network this cause to the sink get disconnected from rest of the network due to its neighbors death [18],[19] and others are still alive and functioning. The mobility of sink is the accepted solution for the energy holes issues in WSNs and further it will increase the network life time. A Mobile Sink Based adaptive Immune Energy Efficient clustering Protocol mitigate the energy hole problem [20]. MSIEEP uses the Adaptive Immune Algorithm (AIA) to find the sojourn locations of the mobile sink and the optimum number of CHs and their locations.

Moreover outperforms the previous protocols in terms of the lifetime, the stability period, the packet drop ratio and the packet delay. In addition to eliminate the energy holes, the control mobile sink that guided based on minimizing the dissipated energy increases the robustness and the ability ofthe proposed protocol to deliver packets to the destination.

\section{Comparision}

The table 1 shows the comparison of various protocols used in Mobile Sink in Wireless Networks. The table does analyze parameter of routing protocol such as pattern architecture, reason to design, problem on existing protocol, and output attained.

\begin{tabular}{|c|c|c|c|c|c|}
\hline Year & $\begin{array}{l}\text { Routing protocols / } \\
\text { algorithms }\end{array}$ & $\begin{array}{ll}\text { Sink } & \text { mobility } \\
\text { pattern } & \\
\end{array}$ & Topology & Reason to design & Output attained \\
\hline 2005 & Mobiroute & Discrete form & flat & $\begin{array}{l}\text { MobiRoute,that } \\
\text { effectively supports sink } \\
\text { mobility.theoretically } \\
\text { proved that moving the } \\
\text { sink can improve network } \\
\text { lifetimer without } \\
\text { sacrificing data delivery } \\
\text { latency. }\end{array}$ & $\begin{array}{l}\text { Improves the network } \\
\text { lifetime with modestly } \\
\text { degraded reliability in } \\
\text { packet delivery. }\end{array}$ \\
\hline 2010 & AVRP & Vorani Cluster & clustered & $\begin{array}{l}\text { A Complete protocol can } \\
\text { increase the network } \\
\text { efficiency while } \\
\text { suppressing the protocol } \\
\text { overhead due to sink } \\
\text { mobility. AVRP design } \\
\text { support the efficient data } \\
\text { gathering in mWSN }\end{array}$ & $\begin{array}{l}\text { Reduce the forwarding } \\
\text { overhead and improve the } \\
\text { packet delivery ratio }\end{array}$ \\
\hline
\end{tabular}


A Survey on Routing Protocols influencing Mobile Sink in enhancing life time of WSN for Data

\begin{tabular}{|c|c|c|c|c|c|}
\hline 2010 & MSRP & $\begin{array}{l}\text { Clustered } \\
\text { Architecture }\end{array}$ & clustered & $\begin{array}{l}\text { This algorithm determines } \\
\text { the best sink path while } \\
\text { each } \mathrm{CH} \text { can freely change } \\
\text { its transmission range (ri) } \\
\text { in order to maximize } \\
\text { network lifetime }\end{array}$ & $\begin{array}{l}\text { Extend the network } \\
\text { lifetime as well as in } \\
\text { improving } \\
\text { throughput }\end{array}$ \\
\hline 2011 & EEMSRA & $\begin{array}{l}\text { Clustered } \\
\text { Architecture }\end{array}$ & clustered & $\begin{array}{l}\text { This algorithm can do } \\
\text { better performance for } \\
\text { energy consumption }\end{array}$ & $\begin{array}{l}\text { Better performance for } \\
\text { energy consumption }\end{array}$ \\
\hline 2013 & HRDG-MS & Random walk & flat & $\begin{array}{l}\text { HRDG-MS protocol is } \\
\text { highly reliable }\end{array}$ & $\begin{array}{l}\text { better performance other } \\
\text { than DDRP }\end{array}$ \\
\hline 2014 & MSREEDG & Random walk & flat & $\begin{array}{l}\text { To control the duplicate } \\
\text { messages are to be } \\
\text { propagated and by } \\
\text { increase the lifetime }\end{array}$ & $\begin{array}{l}\text { increases } \\
\text { both the reliability and } \\
\text { energy efficiency. }\end{array}$ \\
\hline 2015 & MSIEEP & $\begin{array}{l}\text { Clustered } \\
\text { Architecture/ } \\
\text { controlled mobile } \\
\text { sink }\end{array}$ & clustered & $\begin{array}{l}\text { Reduce energy hole } \\
\text { problem and increase the } \\
\text { lifetime }\end{array}$ & $\begin{array}{l}\text { high packet delivery ratio } \\
\text { and low packet drop ratio } \\
\text { compared to the other } \\
\text { protocols }\end{array}$ \\
\hline
\end{tabular}

Table. 1

\section{Conclusion}

As we discussed in this paper, which explain the depiction of mobile sinks with a choice of routing protocols is used in different scenarios to enhance the efficiency and life time of WSN.Asa valuable method, mobile sink nodes incorporated with a wireless sensor network to balance energy consumption. Sink node can move in different ways, such as moving randomly or moving controlled. In this paper, we mainly present an overview and comparison of different circumstances of mobile sink node used to deploy the WSN network with Movable sink node. Moreover, the sink node with routing protocols can be used that can provide better network performance and network lifetime enhancements

\section{References}

[1] I.F.Akyildiz,W.Su,Y.Sankarasubramaniam,E.Cayirci, Wireless sensor networks:asurvey, Elsevier, Computer Networks 38 (2002) 393-422.

[2] J. N. Al-Karaki and A. E. Kamal, "Routing techniques in wireless sensor networks: a survey", IEEE Wireless Communications, vol. 11, December (2004), pp. 6-28.

[3] X. Min, S. Wei-ren, J. Chang-jiang and Z. Ying, "Energy efficient clustering algorithm for maximizing lifetime ofwireless sensor networks", J AEU-International Journalof Electronics and Communications, vol. 64,(2010), pp.2 89-298.

[4] Abbasi, A.A.; Younis, M. A Survey on ClusteringAlgorithms for Wireless Sensor Networks. Computer Network. 2007, 30, 2826-2841.

[5] Suchita R.Wankhade1 and NekitaA.Chavhan, A ReviewOn Data Collection Method With Sink Node In Wireless Sensor Network, International Journal of Distributed andParallel Systems (IJDPS) Vol.4, No.1, January 2013.

[6] Jun Luo Jacques PanchardMichałPi' orkowski, MatthiasGrossglauser Jean-Pierre Hubaux, MobiRoute: Routing towards a Mobile Sink for Improving Lifetime in Sensor Networks, (NCCR-MICS), a center supported by the Swiss National Science Foundation under grant number 5005-67322. (http://www.terminodes.org)

[7] A. Woo, T. Tong, and D. Culler. Taming the Underlying Challenges of Reliable Multihop Routing in Sensor Networks. In Proc. of the 1st ACM SenSys, 2003

[8] KeTian, Baoxian Zhang, Kui Huang and Jian Ma, Data Gathering Protocols for Wireless Sensor Networks with Mobile Sinks, IEEE Globecom 2010 proceedings

[9] H. Dubois-Ferriere and D. Estrin, "Efficient and practical query scoping in sensor networks," in Proc. IEEE MASS'04, 2004.

[10] Babar Nazir, HalabiHasbullah, Mobile Sink based Routing Protocol (MSRP) for Prolonging Network Lifetime in Clustered Wireless Sensor Network, 2010 International Conference on Computer Applications and Industrial Electronics (ICCAIE 2010), December 5-7, 2010, Kuala Lumpur, Malaysia

[11] YUAN Xun-Xin, ZHANG Rui-Hua, An Energy-Efficient Mobile Sink Routing Algorithm for Wireless Sensor Networks, This research is sponsored by the Natural Science Foundation of China (NSFC) under grant no. 60903031 and 61070022, 978-1-42446252-0/11/\$26.00 @ 2011 IEEE.

[12] FarzadTashtarian, Mohammad HosseinYaghmaeeMoghaddam, SohrabEffati, Energy Efficient Data Gathering Algorithm in Hierarchical Wireless Sensor Networks with Mobile Sink, 2012 2nd International eConference on Computer and Knowledge Engineering (ICCKE), October 18-19, 2012

[13] Ji Huang, Danpu Liu, A High-Reliability Data Gathering Protocol Based on Mobile Sinks for Wireless Sensor Networks, 978-14673-5699-2 /13/\$31.00 @2013 IEEE

[14] Reliable Data Gathering by Mobile Sink for Wireless Sensor Networks, International Conference on Communication and Signal Processing, April 3-5, 2014, India

[15] Mohammad HosseinAnisi. Abdul Hanan Abdullah, ShukorAbdRazak,"Energy-efficient data collection in wireless sensor networks", Wireless Sensor Network 3(10) (2011) 329-333, 001: I 0.4236/wsn.20 11.310036.

[16] Junzhao Du, Hui Liu, LongfeiShangguan, Luo Mai, Kai Wang, Shucong Li, "Rendezvous data collection using a mobile element in heterogeneous sensor networks", international Journal of Distributed Sensor Networks 2012 (2012) 12 p, Article 10 686172, DO1: $10.1155 / 2012 / 686172$ 
[17] Mohammad HosseinAnisi, Abdul Hanan Abdullah, ShukorAbdRazak,"Efficient Data Aggregation in Wireless Sensor Networks", Department of Computer Systems and Communications, University Technology Malaysia (UTM), vol. 13, Accession Number: ICFTT2011F097, ISBN- 13: 978-981-08-9916-5, 2011

[18] S. Basagni, A. Carosi, E. Melachrinoudis, C. Petrioli, and Z. M. Wang, "Controlled sink mobility for prolonging wireless sensor networks lifetime," Wireless Netw., vol. 14, no. 6, pp. 831-858, Dec. 2008.

[19] M. R. Jafri, N. Javaid, A. Javaid, and Z. A. Khan, "Maximizing the lifetime of multi-chain PEGASIS using sink mobility," World Appl.Sci. J., vol. 21, no. 9, pp. 1283-1289, Mar. 2013.

[20] Mohammed Abo-Zahhad, Sabah M. Ahmed, Nabil Sabor,andShigenobu Sasaki, "Mobile Sink-Based Adaptive Immune EnergyEfficient Clustering Protocol for Improving the Lifetime and Stability Period of Wireless Sensor Networks" IEEE Sensors Journal, VOL. 15, NO. 8, AUGUST 2015. 